\title{
GENERALIZED ROUGH CESÀRO AND LACUNARY STATISTICAL TRIPLE DIFFERENCE SEQUENCE SPACES IN PROBABILITY OF FRACTIONAL ORDER DEFINED BY MUSIELAK-ORLICZ FUNCTION
}

\author{
A. ESI ${ }^{1}$ AND N. SUBRAMANIAN ${ }^{2, *}$ \\ ${ }^{1}$ Department of Mathematics,Adiyaman University,02040,Adiyaman, Turkey \\ ${ }^{2}$ Department of Mathematics, SASTRA University, Thanjavur-613 401, India \\ ${ }^{*}$ Corresponding author: nsmaths@gmail.com
}

\begin{abstract}
We generalized the concepts in probability of rough Cesàro and lacunary statistical by introducing the difference operator $\Delta_{\gamma}^{\alpha}$ of fractional order, where $\alpha$ is a proper fraction and $\gamma=\left(\gamma_{m n k}\right)$ is any fixed sequence of nonzero real or complex numbers. We study some properties of this operator involving lacunary sequence $\theta$ and arbitrary sequence $p=\left(p_{r s t}\right)$ of strictly positive real numbers and investigate the topological structures of related with triple difference sequence spaces.

The main focus of the present paper is to generalized rough Cesàro and lacunary statistical of triple difference sequence spaces and investigate their topological structures as well as some inclusion concerning the operator $\Delta_{\gamma}^{\alpha}$.
\end{abstract}

\section{INTRODUCTION}

A triple sequence (real or complex) can be defined as a function $x: \mathbb{N} \times \mathbb{N} \times \mathbb{N} \rightarrow \mathbb{R}(\mathbb{C})$, where $\mathbb{N}, \mathbb{R}$ and $\mathbb{C}$ denote the set of natural numbers, real numbers and complex numbers respectively. The different types of notions of triple sequence was introduced and investigated at the initial by Sahiner et al. [10,11], Esi et

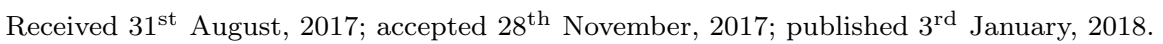

2010 Mathematics Subject Classification. 40F05, 40J05, 40 G05.

Key words and phrases. analytic sequence; Musielak-Orlicz function; triple sequences; chi sequence; Cesàro summable; lacunary statistical.

(C) 2018 Authors retain the copyrights of their papers, and all open access articles are distributed under the terms of the Creative Commons Attribution License. 
al. [1-3], Datta et al. [4],Subramanian et al. [12], Debnath et al. [5] and many others.

A triple sequence $x=\left(x_{m n k}\right)$ is said to be triple analytic if

$$
\sup _{m, n, k}\left|x_{m n k}\right|^{\frac{1}{m+n+k}}<\infty \text {. }
$$

The space of all triple analytic sequences are usually denoted by $\Lambda^{3}$. A triple sequence $x=\left(x_{m n k}\right)$ is called triple gai sequence if

$$
\left((m+n+k) !\left|x_{m n k}\right|\right)^{\frac{1}{m+n+k}} \rightarrow 0 \text { as } m, n, k \rightarrow \infty .
$$

The notion of difference sequence spaces (for single sequences) was introduced by Kizmaz [6] as follows

$$
Z(\Delta)=\left\{x=\left(x_{k}\right) \in w:\left(\Delta x_{k}\right) \in Z\right\}
$$

for $Z=c, c_{0}$ and $\ell_{\infty}$, where $\Delta x_{k}=x_{k}-x_{k+1}$ for all $k \in \mathbb{N}$.

The difference triple sequence space was introduced by Debnath et al. (see [5]) and is defined as $\Delta x_{m n k}=x_{m n k}-x_{m, n+1, k}-x_{m, n, k+1}+x_{m, n+1, k+1}-x_{m+1, n, k}+x_{m+1, n+1, k}+x_{m+1, n, k+1}-x_{m+1, n+1, k+1}$ and $\Delta^{0} x_{m n k}=\left\langle x_{m n k}\right\rangle$.

\section{Some NEW DIFFEREnCE TRIPLE SEQUENCE SPACES WiTH FRACTIONAL ORDER}

Let $\Gamma(\alpha)$ denote the Euler gamma function of a real number $\alpha$. Using the definition $\Gamma(\alpha)$ with $\alpha \notin$ $\{0,-1,-2,-3, \cdots\}$ cab be expressed as an improper integral as follows: $\Gamma(\alpha)=\int_{0}^{\infty} e^{-x} x^{\alpha-1} d x$, where $\alpha$ is a positive proper fraction. We have defined the generalized fractional triple sequence spaces of difference operator

$$
\Delta_{\gamma}^{\alpha}\left(x_{m n k}\right)=\sum_{u=0}^{\infty} \sum_{v=0}^{\infty} \sum_{w=0}^{\infty} \frac{(-1)^{u+v+w} \Gamma(\alpha+1)}{(u+v+w) ! \Gamma(\alpha-(u+v+w)+1)} x_{m+u, n+v, k+w} .
$$

In particular, we have

(i) $\Delta^{\frac{1}{2}}\left(x_{m n k}\right)=x_{m n k}-\frac{1}{16} x_{m+1, n+1, k+1}-\cdots$.

(ii) $\Delta^{-\frac{1}{2}}\left(x_{m n k}\right)=x_{m n k}+\frac{5}{16} x_{m+1, n+1, k+1}+\cdots$.

(iii) $\Delta^{\frac{2}{3}}\left(x_{m n k}\right)=x_{m n k}-\frac{4}{81} x_{m+1, n+1, k+1}-\cdots$. Now we determine the new classes of triple difference sequence spaces $\Delta_{\gamma}^{\alpha}(x)$ as follows:

$$
\Delta_{\gamma}^{\alpha}(x)=\left\{x:\left(x_{m n k}\right) \in w^{3}:\left(\Delta_{\gamma}^{\alpha} x\right) \in X\right\}
$$

where $\Delta_{\gamma}^{\alpha}\left(x_{m n k}\right)=\sum_{u=0}^{\infty} \sum_{v=0}^{\infty} \sum_{w=0}^{\infty} \frac{(-1)^{u+v+w} \Gamma(\alpha+1)}{(u+v+w) ! \Gamma(\alpha-(u+v+w)+1)} x_{m+u, n+v, k+w}$ and

$$
X \in \chi_{f}^{3 \Delta}(x)=\chi_{f}^{3}\left(\Delta_{\gamma}^{\alpha} x_{m n k}\right)=\mu_{m n k}\left(\Delta_{\gamma}^{\alpha} x\right)=\left[f_{m n k}\left(\left((m+n+k) !\left|\Delta_{\gamma}^{\alpha}\right|\right)^{\frac{1}{m+n+k}}, \overline{0}\right)\right]
$$

Proposition 2.1. (i) For a proper fraction $\alpha, \quad \Delta^{\alpha}: W \times W \times W \rightarrow W \times W \times W$ defined by equation of (2.1) is a linear operator. 
(ii) For $\alpha, \beta>0, \Delta^{\alpha}\left(\Delta^{\beta}\left(x_{m n k}\right)\right)=\Delta^{\alpha+\beta}\left(x_{m n k}\right)$ and $\Delta^{\alpha}\left(\Delta^{-\alpha}\left(x_{m n k}\right)\right)=x_{m n k}$.

\section{Proof: Omitted.}

Proposition 2.2. For a proper fraction $\alpha$ and $f$ be an Musielak-Orlicz function, if $\chi_{f}^{3}(x)$ is a linear space, then $\chi_{f}^{3 \Delta_{\gamma}^{\alpha}}(x)$ is also a linear space.

Proof: Omitted

\section{Definitions and Preliminaries}

Throughout the article $w^{3}, \chi^{3}(\Delta), \Lambda^{3}(\Delta)$ denote the spaces of all, triple gai difference sequence spaces and triple analytic difference sequence spaces respectively.

Subramanian et al. (see [12]) introduced by a triple entire sequence spaces, triple analytic sequences spaces and triple gai sequence spaces. The triple sequence spaces of $\chi^{3}(\Delta), \Lambda^{3}(\Delta)$ are defined as follows:

$$
\begin{aligned}
& \chi^{3}(\Delta)=\left\{x \in w^{3}:\left((m+n+k) !\left|\Delta x_{m n k}\right|\right)^{1 / m+n+k} \rightarrow 0 \text { as } m, n, k \rightarrow \infty\right\}, \\
& \Lambda^{3}(\Delta)=\left\{x \in w^{3}: \sup _{m, n, k}\left|\Delta x_{m n k}\right|^{1 / m+n+k}<\infty\right\} .
\end{aligned}
$$

Definition 3.1. An Orlicz function ([see [7]) is a function $M:[0, \infty) \rightarrow[0, \infty)$ which is continuous, nondecreasing and convex with $M(0)=0, M(x)>0$, for $x>0$ and $M(x) \rightarrow \infty$ as $x \rightarrow \infty$. If convexity of Orlicz function $M$ is replaced by $M(x+y) \leq M(x)+M(y)$, then this function is called modulus function.

Lindenstrauss and Tzafriri ([8]) used the idea of Orlicz function to construct Orlicz sequence space.

A sequence $g=\left(g_{m n}\right)$ defined by

$$
g_{m n}(v)=\sup \left\{|v| u-\left(f_{m n k}\right)(u): u \geq 0\right\}, m, n, k=1,2, \cdots
$$

is called the complementary function of a Musielak-Orlicz function $f$. For a given Musielak-Orlicz function $f$, [see [9] ] the Musielak-Orlicz sequence space $t_{f}$ is defined as follows

$$
t_{f}=\left\{x \in w^{3}: I_{f}\left(\left|x_{m n k}\right|\right)^{1 / m+n+k} \rightarrow 0 \text { as } m, n, k \rightarrow \infty\right\},
$$

where $I_{f}$ is a convex modular defined by

$$
I_{f}(x)=\sum_{m=1}^{\infty} \sum_{n=1}^{\infty} \sum_{k=1}^{\infty} f_{m n k}\left(\left|x_{m n k}\right|\right)^{1 / m+n+k}, x=\left(x_{m n k}\right) \in t_{f} .
$$

We consider $t_{f}$ equipped with the Luxemburg metric

$$
d(x, y)=\sum_{m=1}^{\infty} \sum_{n=1}^{\infty} \sum_{k=1}^{\infty} f_{m n k}\left(\frac{\left|x_{m n k}\right|^{1 / m+n+k}}{m n k}\right)
$$

is an exteneded real number.

Definition 3.2. Let $\alpha$ be a proper fraction. A triple difference sequence spaces of $\Delta_{\gamma}^{\alpha} x=\left(\Delta_{\gamma}^{\alpha} x_{m n k}\right)$ is said to be $\Delta_{\gamma}^{\alpha}$ strong Cesàro summable to $\overline{0}$ if 
$\lim _{u v w \rightarrow \infty} \frac{1}{u v w} \sum_{m=1}^{u} \sum_{n=1}^{v} \sum_{k=1}^{w}\left|\Delta_{\gamma}^{\alpha} x_{m n k}, \overline{0}\right|=0$. In this we write $\Delta_{\gamma}^{\alpha} x_{m n k} \rightarrow^{[C, 1,1,1]} \Delta_{\gamma}^{\alpha} x_{m n k}$. The set of all $\Delta_{\gamma}^{\alpha}$ strong Cesàro summable triple sequence spaces is denoted by $[C, 1,1,1]$.

Definition 3.3. Let $\alpha$ be a proper fraction and $\beta$ be a nonnegative real number. A triple difference sequence spaces of $\Delta_{\gamma}^{\alpha} x=\left(\Delta_{\gamma}^{\alpha} x_{m n k}\right)$ is said to be $\Delta_{\gamma}^{\alpha}$ rough strong Cesàro summable in probability to a random variable $\Delta_{\gamma}^{\alpha} x: W \times W \times W \rightarrow \mathbb{R} \times \mathbb{R} \times \mathbb{R}$ with respect to the roughness of degree $\beta$ if for each $\epsilon>0$,

$\lim _{u v w \rightarrow \infty} \frac{1}{u v w} \sum_{m=1}^{u} \sum_{n=1}^{v} \sum_{k=1}^{w} P\left(\left|\Delta_{\gamma}^{\alpha} x_{m n k}, \overline{0}\right| \geq \beta+\epsilon\right)=0$. In this case we write $\Delta_{\gamma}^{\alpha} x_{m n k} \rightarrow{ }_{\beta}^{[C, 1,1,1]^{P \Delta}}$ $\Delta_{\gamma}^{\alpha} x_{m n k}$. The class of all $\beta \Delta_{\gamma}^{\alpha}-$ strong Cesàro summable triple sequence spaces of random variables in proability and it will be denoted by $\beta[C, 1,1,1]^{P \Delta}$.

\section{Rough Ces $\grave{A}$ Ro summable of triple of $\Delta_{\gamma}^{\alpha}$}

In this section by using the operator $\Delta_{\gamma}^{\alpha}$, we introduce some new triple difference sequence spaces of rough Cesàro summable involving lacunary sequences $\theta$ and arbitrary sequence $p=\left(p_{\text {rst }}\right)$ of strictly positive real numbers.

If $\alpha$ be a proper fraction and $\beta$ be nonnegative real number. $\mathrm{A}$ triple difference sequence spaces of $\Delta_{\gamma}^{\alpha} X=\left(\Delta_{\gamma}^{\alpha} x_{m n k}\right)$ is said to be $\Delta_{\gamma}^{\alpha}$ - rough strong Cesàro summable in probability to a random variable $\Delta_{\gamma}^{\alpha} X: W \times W \times W \rightarrow \mathbb{R} \times \mathbb{R} \times \mathbb{R}$ with respect to the roughness of degree $\beta$ if for each $\epsilon>0$ then define the triple difference sequence spaces as follows: (i)

$C\left(\Delta_{\gamma}^{\alpha}, p\right)_{\theta}=\sum_{r=1}^{\infty} \sum_{s=1}^{\infty} \sum_{t=1}^{\infty} P\left(f_{m n k}\left[\left|\frac{1}{h_{r s t}} \sum_{(m n k) \in I_{r s t}} \Delta_{\gamma}^{\alpha} X\right|^{p_{r s t}}\right] \geq \beta+\epsilon\right)<\infty$. In this case we write $C\left(\Delta_{\gamma}^{\alpha}, p\right)_{\theta} \rightarrow_{\beta}^{[C, 1,1,1]^{P \Delta}} C\left(\Delta_{\gamma}^{\alpha}, p\right)_{\theta}$. The class of all $\beta C\left(\Delta_{\gamma}^{\alpha}, p\right)_{\theta}-$ rough strong Cesàro summable triple sequence spaces of random variables in probability and it will be denoted by $\beta[C, 1,1,1]^{P \Delta}$.

$C\left[\Delta_{\gamma}^{\alpha}, p\right]_{\theta}=\sum_{r=1}^{\infty} \sum_{s=1}^{\infty} \sum_{t=1}^{\infty} P\left(\frac{1}{h_{r s t}} \sum_{(m n k) \in I_{r s t}} f_{m n k}\left[\left|\Delta_{\gamma}^{\alpha} X\right|^{p_{r s t}}\right] \geq \beta+\epsilon\right)<\infty$. In this case we write $C\left[\Delta_{\gamma}^{\alpha}, p\right]_{\theta} \rightarrow_{\beta}^{[C, 1,1,1]^{P \Delta}} C\left[\Delta_{\gamma}^{\alpha}, p\right]_{\theta}$. The class of all $\beta C\left[\Delta_{\gamma}^{\alpha}, p\right]_{\theta}$ - rough strong Cesàro summable triple sequence spaces of random variables in probability.

(iii)

$C_{\Lambda}\left(\Delta_{\gamma}^{\alpha}, p\right)_{\theta}=P\left(f_{m n k}\left[\left|\frac{1}{h_{r s t}} \sum_{(m n k) \in I_{r s t}} \Delta_{\gamma}^{\alpha} X\right|^{p_{r s t}}\right] \geq \beta+\epsilon\right)<\infty$. In this case we write $C_{\Lambda}\left(\Delta_{\gamma}^{\alpha}, p\right)_{\theta} \rightarrow_{\beta}^{[C, 1,1,1]^{P \Delta}}$ $C_{\Lambda}\left(\Delta_{\gamma}^{\alpha}, p\right)_{\theta}$. The class of all $\beta C_{\Lambda}\left(\Delta_{\gamma}^{\alpha}, p\right)_{\theta}$ - rough strong Cesàro summable triple sequence spaces of random variables in probability.

$C_{\Lambda}\left[\Delta_{\gamma}^{\alpha}, p\right]_{\theta}=\frac{1}{h_{r s t}} \sum_{(m n k) \in I_{r s t}} P\left(f_{m n k}\left[\left|\Delta_{\gamma}^{\alpha} X\right|^{p_{r s t}}\right] \geq \beta+\epsilon\right)<\infty$. In this case we write $C_{\Lambda}\left[\Delta_{\gamma}^{\alpha}, p\right]_{\theta} \rightarrow{ }_{\beta}^{[C, 1,1,1]^{P \Delta}}$ $C_{\Lambda}\left[\Delta_{\gamma}^{\alpha}, p\right]_{\theta}$. The class of all $\beta C_{\Lambda}\left[\Delta_{\gamma}^{\alpha}, p\right]_{\theta}$ - rough strong Cesàro summable triple sequence spaces of random variables in probability.

$N\left(\Delta_{\gamma}^{\alpha}, p\right)_{\theta}=\lim _{r s t \rightarrow \infty} \frac{1}{h_{r s t}} \sum_{(m n k) \in I_{r s t}} P\left(f_{m n k}\left[\left|\Delta_{\gamma}^{\alpha} X, \overline{0}\right|^{p_{r s t}}\right] \geq \beta+\epsilon\right)=0$. In this case we write $N\left(\Delta_{\gamma}^{\alpha}, p\right)_{\theta} \rightarrow{ }_{\beta}^{[C, 1,1,1]^{P \Delta}}$ 
$N\left(\Delta_{\gamma}^{\alpha}, p\right)_{\theta}$. The class of all $\beta N\left(\Delta_{\gamma}^{\alpha}, p\right)_{\theta}$ - rough strong Cesàro summable triple sequence spaces of random variables in probability.

Theorem 4.1. If $\alpha$ be a proper fraction, $\beta$ be nonnegative real number, $f$ be an Musielak-Orlicz function and $\left(p_{\text {rst }}\right)$ is a triple difference analytic sequence then the sequence spaces $C\left(\Delta_{\gamma}^{\alpha}, p\right)_{\theta}, C\left[\Delta_{\gamma}^{\alpha}, p\right]_{\theta}, C_{\Lambda}\left(\Delta_{\gamma}^{\alpha}, p\right)_{\theta}$, $C_{\Lambda}\left[\Delta_{\gamma}^{\alpha}, p\right]_{\theta}$ and $N\left(\Delta_{\gamma}^{\alpha}, p\right)_{\theta}$ are linear spaces.

Proof: Because the linearity may be proved in a similar way for each of the sets of triple sequences, hence it is omitted.

Theorem 4.2. If $\alpha$ be a proper fraction, $\beta$ be nonnegative real number, $f$ be an Musielak-Orlicz function and $\left(p_{r s t}\right)$, for all $r, s, t \in \mathbb{N}$, then the triple difference sequence spaces $C\left[\Delta_{\gamma}^{\alpha}, p\right]_{\theta}$ is a BK-space with the luxemburg metric is defined by

$d(x, y)_{1}=\sum_{u=0}^{\infty} \sum_{v=0}^{\infty} \sum_{w=0}^{\infty} f_{m n k}\left[\frac{\gamma_{u v w} x_{u v w}}{u v w}\right]+$

$\lim _{u v w \rightarrow \infty} \frac{1}{u v w} \sum_{r=1}^{\infty} \sum_{s=1}^{\infty} \sum_{t=1}^{\infty} f_{m n k}\left[P\left(\frac{1}{h_{r s t}} \sum_{(m, n, k) \in I_{r s t}}\left|\Delta_{\gamma}^{\alpha} x\right|^{p}\right) \geq \beta+\epsilon\right]^{1 / p}, 1 \leq p$.

Also if $p_{\text {rst }}=1$ for all $(r, s, t) \in \mathbb{N}$, then the triple difference spaces $C_{\Lambda}\left[\Delta_{\gamma}^{\alpha}, p\right]_{\theta}$ and $N\left(\Delta_{\gamma}^{\alpha}, p\right)_{\theta}$ are $B K$ spaces with the luxemburg metric is defined by

$d(x, y)_{2}=\sum_{u=0}^{\infty} \sum_{v=0}^{\infty} \sum_{w=0}^{\infty} f_{m n k}\left[\frac{\gamma_{u v w} x_{u v w}}{u v w}\right]+$

$\lim _{u v w \rightarrow \infty} \frac{1}{u v w} \frac{1}{h_{r s t}} \sum_{(m, n, k) \in I_{r s t}} f_{m n k}\left[P\left(\left|\Delta_{\gamma}^{\alpha} x\right|\right) \geq \beta+\epsilon\right]$.

Proof: We give the proof for the space $C_{\Lambda}\left[\Delta_{\gamma}^{\alpha}, p\right]_{\theta}$ and that of others followed by using similar techniques. Suppose $\left(x^{n}\right)$ is a Cauchy sequence in $C_{\Lambda}\left[\Delta_{\gamma}^{\alpha}, p\right]_{\theta}$, where $x^{n}=\left(x_{i j \ell}\right)^{n}$ and $x^{m}=\left(x_{i j \ell}^{m}\right)$ are two elements in $C_{\Lambda}\left[\Delta_{\gamma}^{\alpha}, p\right]_{\theta}$. Then there exists a positive integer $n_{0}(\epsilon)$ such that $\left|x^{n}-x^{m}\right| \rightarrow 0$ as $m, n \rightarrow \infty$. for all $m, n \geq n_{0}(\epsilon)$ and for each $i, j, \ell \in \mathbb{N}$. Therefore

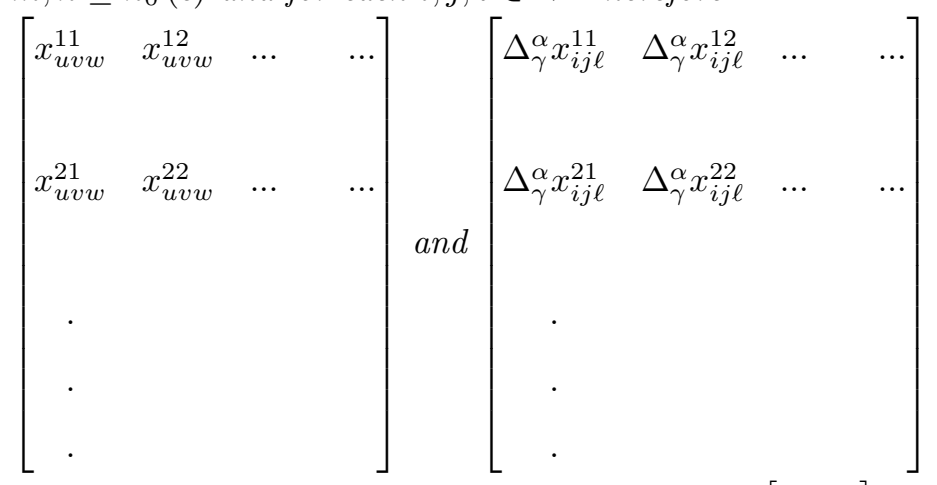

are Cauchy sequences in complex field $\mathbb{C}$ and $C_{\Lambda}\left[\Delta_{\gamma}^{\alpha}, p\right]_{\theta}$ respectively. By using the completness of $\mathbb{C}$ and $C_{\Lambda}\left[\Delta_{\gamma}^{\alpha}, p\right]_{\theta}$ we have that they are convergent and suppose that $x_{i j \ell}^{n} \rightarrow x_{i j \ell}$ in $\mathbb{C}$ and $\left(\Delta_{\gamma}^{\alpha} x_{i j \ell}^{n}\right) \rightarrow y_{i j \ell}$ in $C_{\Lambda}\left[\Delta_{\gamma}^{\alpha}, p\right]_{\theta}$ for each $i, j, \ell \in \mathbb{N}$ as $n \rightarrow \infty$. Then we can find a triple sequence space of $\left(x_{i j \ell}\right)$ such that $y_{i j \ell}=\Delta_{\gamma}^{\alpha} x_{i j \ell}$ for $i, j, \ell \in \mathbb{N}$. These $x_{i j \ell}^{s}$ can be interpreted as

$x_{i j \ell}=\frac{1}{\gamma_{i j \ell}} \sum_{u=1}^{i-m} \sum_{v=1}^{j-n} \sum_{w=1}^{\ell-k} \Delta_{\gamma}^{\alpha} y_{u v w}=$ $\frac{1}{\gamma_{i j \ell}} \sum_{u=1}^{i} \sum_{v=1}^{j} \sum_{w=1}^{\ell} \Delta_{\gamma}^{\alpha} y_{u-m, v-n, w-k},\left(y_{1-m, 1-n, 1-k}=y_{2-m, 2-n, 2-k}=\cdots=y_{000}=0\right)$. for sufficiently large $(i, j, \ell)$; that is, 
$\left(\Delta_{\gamma}^{\alpha} x^{n}\right)=\left[\begin{array}{cccc}\Delta_{\gamma}^{\alpha} x_{i j \ell}^{11} & \Delta_{\gamma}^{\alpha} x_{i j \ell}^{12} & \ldots & \ldots \\ \Delta_{\gamma}^{\alpha} x_{i j \ell}^{21} & \Delta_{\gamma}^{\alpha} x_{i j \ell}^{22} & \ldots & \ldots \\ \cdot & & & \\ \cdot & & & \\ \cdot & & & \end{array}\right]$

converges to $\left(\Delta_{\gamma}^{\alpha} x_{i j \ell}\right)$ for each $i, j, \ell \in \mathbb{N}$ as $n \rightarrow \infty$. Thus $\left|x^{m}-x\right|_{2} \rightarrow 0$ as $m \rightarrow \infty$. Since $C_{\Lambda}\left[\Delta_{\gamma}^{\alpha}, p\right]_{\theta}$ is a Banach luxemburg metric with continuous coordinates, that is $\left|x^{n}-x\right|_{2} \rightarrow 0$ implies $\left|x_{i j \ell}^{n}-x_{i j \ell}\right| \rightarrow 0$ for each $i, j, \ell \in \mathbb{N}$ as $n \rightarrow \infty$, this shows that $C_{\Lambda}\left[\Delta_{\gamma}^{\alpha}, p\right]_{\theta}$ is a BK-space.

Theorem 4.3. If $\alpha$ be a proper fraction, $\beta$ be nonnegative real number, $f$ be an Musielak-Orlicz function and $\left(p_{r s t}\right)$, for all $r, s, t \in \mathbb{N}$, then the triple difference sequence space $C\left(\Delta_{\gamma}^{\alpha}, p\right)_{\theta}$ is a BK-space with the luxemburg metric is defined by

$d(x, y)_{3}=\sum_{u=0}^{\infty} \sum_{v=0}^{\infty} \sum_{w=0}^{\infty} f_{m n k}\left[\frac{\gamma_{u v w} x_{u v w}}{u v w}\right]+$ $\lim _{u v w \rightarrow \infty} \frac{1}{u v w} \sum_{r=1}^{\infty} \sum_{s=1}^{\infty} \sum_{t=1}^{\infty} f_{m n k}\left[P\left(\left|\frac{1}{h_{r s t}} \sum_{(m, n, k) \in I_{r s t}} \Delta_{\gamma}^{\alpha} x\right|^{p}\right) \geq \beta+\epsilon\right]^{1 / p}, 1 \leq p$.

Also if $p_{\text {rst }}=1$ for all $(r, s, t) \in \mathbb{N}$, then the triple difference spaces $C_{\Lambda}\left(\Delta_{\gamma}^{\alpha}, p\right)_{\theta}$ is a BK-spaces with the luxemburg metric is defined by

$d(x, y)_{4}=\sum_{u=0}^{\infty} \sum_{v=0}^{\infty} \sum_{w=0}^{\infty} f_{m n k}\left[\frac{\gamma_{u v w} X_{u v w}}{u v w}\right]+$ $\lim _{u v w \rightarrow \infty} \frac{1}{u v w} f_{m n k}\left[P\left(\left|\frac{1}{h_{r s t}} \sum_{(m, n, k) \in I_{r s t}} \Delta_{\gamma}^{\alpha} x\right|\right) \geq \beta+\epsilon\right]$.

Proof: The proof follows from Theorem 4.2.

Now, we can present the following theorem, determining some inclusion relations with out proof, since it is a routine verification.

Theorem 4.4. Let $\alpha, \xi$ be two positive proper fractions $\alpha>\xi>0$ and $\beta$ be two nonnegative real number, $f$ be an Musielak-Orlicz function and $\left(p_{r s t}\right)=p$, for each $r, s, t \in \mathbb{N}$ be given. Then the following inclusions are satisfied:

(i) $C\left(\Delta_{\gamma}^{\xi}, p\right)_{\theta} \subset C\left(\Delta_{\gamma}^{\alpha}, p\right)_{\theta}$,

(ii) $C\left[\Delta_{\gamma}^{\xi}, p\right]_{\theta} \subset C\left[\Delta_{\gamma}^{\alpha}, p\right]_{\theta}$,

(i) $C\left(\Delta_{\gamma}^{\alpha}, p\right)_{\theta} \subset C\left(\Delta_{\gamma}^{\alpha}, q\right)_{\theta}, 0<p<q$.

\section{Rough Lacunary Statistical CONVERgence of triple of $\Delta_{\gamma}^{\alpha}$}

In this section by using the operator $\Delta_{\gamma}^{\alpha}$, we introduce some new triple difference sequence spaces involving rough lacunary statistical sequences spaces and arbitrary sequence $p=\left(p_{r s t}\right)$ of strictly positive real numbers. 
Definition 5.1. The triple sequence $\theta_{i, \ell, j}=\left\{\left(m_{i}, n_{\ell}, k_{j}\right)\right\}$ is called triple lacunary if there exist three increasing sequences of integers such that

$$
\begin{gathered}
m_{0}=0, h_{i}=m_{i}-m_{r-1} \rightarrow \infty \text { as } i \rightarrow \infty \text { and } \\
n_{0}=0, \overline{h_{\ell}}=n_{\ell}-n_{\ell-1} \rightarrow \infty \text { as } \ell \rightarrow \infty . \\
k_{0}=0, \overline{h_{j}}=k_{j}-k_{j-1} \rightarrow \infty \text { as } j \rightarrow \infty .
\end{gathered}
$$

Let $m_{i, \ell, j}=m_{i} n_{\ell} k_{j}, h_{i, \ell, j}=h_{i} \overline{h_{\ell} h_{j}}$, and $\theta_{i, \ell, j}$ is determine by

$I_{i, \ell, j}=\left\{(m, n, k): m_{i-1}<m<m_{i}\right.$ and $n_{\ell-1}<n \leq n_{\ell}$ and $\left.k_{j-1}<k \leq k_{j}\right\}, q_{i}=\frac{m_{i}}{m_{i-1}}, \overline{q_{\ell}}=\frac{n_{\ell}}{n_{\ell-1}}, \overline{q_{j}}=\frac{k_{j}}{k_{j-1}}$.

Definition 5.2. Let $\alpha$ be a proper fraction, $f$ be an Musielak-Orlicz function and $\theta=\left\{m_{r} n_{s} k_{t}\right\}_{(r s t) \in \mathbb{N} \cup 0}$ be the triple difference lacunary sequence spaces of $\left(\Delta_{\gamma}^{\alpha} X_{m n k}\right)$ is said to be $\Delta_{\gamma}^{\alpha}$ - lacunary statistically convergent to a number $\overline{0}$ if for any $\epsilon>0$,

$\lim _{r s t \rightarrow \infty} \frac{1}{h_{r s t}}\left|\left\{(m, n, k) \in I_{r s t}: f_{m n k}\left[\left|\Delta_{\gamma}^{\alpha} X_{m n k}, \overline{0}\right|\right] \geq \epsilon\right\}\right|=0$, where

$I_{r, s, t}=\left\{(m, n, k): m_{r-1}<m<m_{r}\right.$ and $n_{s-1}<n \leq n_{s}$ and $\left.k_{t-1}<k \leq k_{t}\right\}, q_{r}=\frac{m_{r}}{m_{r-1}}, \overline{q_{s}}=\frac{n_{s}}{n_{s-1}}, \overline{q_{t}}=\frac{k_{t}}{k_{t-1}}$.

In this case write $\Delta_{\gamma}^{\alpha} X \rightarrow^{S_{\theta}} \Delta_{\gamma}^{\alpha} x$.

Definition 5.3. If $\alpha$ be a proper fraction, $\beta$ be nonnegative real number, $f$ be an Musielak-Orlicz function and $\theta=\left\{m_{r} n_{s} k_{t}\right\}_{(r s t) \in \mathbb{N} \cup 0}$ be the triple difference sequence spaces of lacunary. A number $X$ is said to be $\Delta_{\gamma}^{\alpha}-N_{\theta}-$ convergent to a real number $\overline{0}$ if for every $\epsilon>0$,

$\lim _{r s t \rightarrow \infty} \frac{1}{h_{r s t}} \sum_{m \in I_{r}} \sum_{n \in I_{s}} \sum_{k \in I_{t}} f_{m n k}\left[\left|\Delta_{\gamma}^{\alpha} X_{m n k}, \overline{0}\right|\right]=0$. In this case we write $\Delta_{\gamma}^{\alpha} X_{m n k} \rightarrow^{N_{\theta}} \overline{0}$.

Definition 5.4. Let $\alpha$ be a proper fraction, $\beta$ be nonnegative real number, $f$ be an Musielak-Orlicz function and arbitary sequence $p=\left(p_{\text {rst }}\right)$ of strictly positive real numbers. A triple difference sequence spaces of random variables is said to be $\Delta_{\gamma}^{\alpha}-$ rough lacunary statistically convergent in probability to $\Delta_{\gamma}^{\alpha} X: W \times W \times$ $W \rightarrow \mathbb{R} \times \mathbb{R} \times \mathbb{R}$ with respect to the roughness of degree $\beta$ if for any $\epsilon, \delta>0$, lim $_{\text {rst } \rightarrow \infty}$

$\frac{1}{h_{r s t}}\left|\left\{(m, n, k) \in I_{r s t}: P\left(\left[f_{m n k}\left(\left|\Delta_{\gamma}^{\alpha}\left(x_{m n k}\right)\right|\right)\right]^{p_{r s t}} \geq \beta+\epsilon\right) \geq \delta\right\}\right|=0$ and we write $\Delta_{\gamma}^{\alpha} X_{m n k} \rightarrow S_{\beta}^{P} \overline{0}$. It will be denoted by $\beta S_{\theta}^{P}$.

Definition 5.5. Let $\alpha$ be a proper fraction, $\beta$ be nonnegative real number, $f$ be an Musielak-Orlicz function and arbitary sequence $p=\left(p_{\text {rst }}\right)$ of strictly positive real numbers. A triple difference sequence spaces of random variables is said to be $\Delta_{\gamma}^{\alpha}-$ rough $N_{\theta}$ - convergent in probability to $\Delta_{\gamma}^{\alpha} X: W \times W \times W \rightarrow \mathbb{R} \times \mathbb{R} \times \mathbb{R}$ with respect to the roughness of degree $\beta$ if for any $\epsilon>0, \lim _{r s t \rightarrow \infty} \frac{1}{h_{r s t}} \sum_{m \in I_{r}} \sum_{n \in I_{s}} \sum_{k \in I_{t}}$ $\left|\left\{P\left(\left[f_{m n k}\left(\left|\Delta_{\gamma}^{\alpha} X_{m n k}\right|\right)\right]^{p_{r s t}} \geq \beta+\epsilon\right)\right\}\right|=0$, and we write $\Delta_{\gamma}^{\alpha} X_{m n k} \rightarrow_{\beta}^{N_{\theta}^{P}} \Delta_{\gamma}^{\alpha} X$. The class of all $\beta-N_{\theta}-$ convergent triple difference sequence spaces of random variables in probability will be denoted by $\beta N_{\theta}^{P}$.

Definition 5.6. Let $\alpha$ be a proper fraction, $\beta$ be nonnegative real number, $f$ be an Musielak-Orlicz function and arbitary sequence $p=\left(p_{\text {rst }}\right)$ of strictly positive real numbers. A triple difference sequence spaces of random variables is said to be $\Delta_{\gamma}^{\alpha}-$ rough lacunary statistically Cauchy if there exists a number $N=N(\epsilon)$ 
in probability to $\Delta_{\gamma}^{\alpha} X: W \times W \times W \rightarrow \mathbb{R} \times \mathbb{R} \times \mathbb{R}$ with respect to the roughness of degree $\beta$ if for any $\epsilon, \delta>0$, $\lim _{r s t \rightarrow \infty}$

$\frac{1}{h_{r s t}}\left|\left\{(m, n, k) \in I_{r s t}: P\left(\left[f_{m n k}\left(\left|\Delta_{\gamma}^{\alpha}\left(x_{m n k}-x_{N}\right)\right|\right)\right]^{p_{r s t}} \geq \beta+\epsilon\right) \geq \delta\right\}\right|=0$.

Theorem 5.1. Let $\alpha$ be a proper fraction, $\beta$ be nonnegative real number, $f$ be an Musielak-Orlicz function and arbitary sequence $p=\left(p_{\text {rst }}\right)$ of strictly positive real numbers, $0<p<\infty$. (i) If $\left(x_{m n k}\right) \rightarrow\left(N\left(\Delta_{\gamma}^{\alpha}, p\right)_{\theta}\right)$ for $p_{\text {rst }}=p$ then $\left(x_{m n k}\right) \rightarrow\left(\Delta_{\gamma}^{\alpha}\left(S_{\theta}\right)\right)$. (ii) If $x \in\left(\Delta_{\gamma}^{\alpha}\left(S_{\theta}\right)\right)$, then $\left(x_{m n k}\right) \rightarrow\left(N\left(\Delta_{\gamma}^{\alpha}, p\right)_{\theta}\right)$.

Proof: Let $x=\left(x_{m n k}\right) \in\left(N\left(\Delta_{\gamma}^{\alpha}, p\right)_{\theta}\right)$ and $\epsilon>0,\left|\left\{P\left(\left[f_{m n k}\left(\left|\Delta_{\gamma}^{\alpha} X_{m n k}\right|\right)\right]^{p_{r s t}} \geq \beta+\epsilon\right)\right\}\right|=0$. We have $\frac{1}{h_{r s t}} \sum_{(m n k) \in I_{r s t}}\left|\left\{P\left(\left[f_{m n k}\left(\left|\Delta_{\gamma}^{\alpha} X_{m n k}\right|\right)\right]^{p_{r s t}} \geq \beta+\epsilon\right)\right\}\right| \geq$ $\frac{1}{h_{r s t}}\left|\left\{(m, n, k) \in I_{r s t}: P\left(\left[f_{m n k}\left(\left|\Delta_{\gamma}^{\alpha}\left(x_{m n k}\right)\right|\right)\right]^{p_{r s t}} \geq \beta+\epsilon\right) \geq \delta\right\}\right|\left(\frac{\beta+\epsilon}{\delta}\right)^{p}$.

So we observe by passing to limit as $r, s, t \rightarrow \infty$,

$\lim _{r s t \rightarrow \infty} \frac{1}{h_{r s t}}\left|\left\{(m, n, k) \in I_{r s t}: P\left(\left[f_{m n k}\left(\left|\Delta_{\gamma}^{\alpha}\left(x_{m n k}\right)\right|\right)\right]^{p_{r s t}} \geq \beta+\epsilon\right) \geq \delta\right\}\right| \leq$ $\left(\frac{\delta}{\alpha+\epsilon}\right)^{p} P\left(\lim _{r s t \rightarrow \infty} \frac{1}{h_{r s t}} \sum_{(m, n, k) \in I_{r s t}}\left|\Delta_{\gamma}^{\alpha} x_{m n k}\right|^{p}\right)=0$. which implies that $x_{m n k} \rightarrow\left(\Delta_{\gamma}^{\alpha}\left(S_{\theta}\right)\right)$.

Suppose that $x \in \Delta_{\gamma}^{\alpha}\left(\Lambda^{3}\right)$ and $\left(x_{m n k}\right) \rightarrow\left(\Delta_{\gamma}^{\alpha}(S)\right)$. Then it is obvious that $\left(\Delta_{\gamma}^{\alpha} x\right) \in \Lambda^{3}$ and $\frac{1}{h_{r s t}}\left|\left\{(m, n, k) \in I_{r s t}: P\left(\left[f_{m n k}\left(\left|\Delta_{\gamma}^{\alpha}\left(x_{m n k}\right)\right|\right)\right]^{p_{r s t}} \geq \beta+\epsilon\right) \geq \delta\right\}\right| \rightarrow 0$ as $r, s, t \rightarrow \infty$. Let $\epsilon>0$ be given and there exists $u_{0} v_{0} w_{0} \in \mathbb{N}$ such that

$\left|\left\{(m, n, k) \in I_{r s t}: P\left(\left[f_{m n k}\left(\left|\Delta_{\gamma}^{\alpha}\left(x_{m n k}\right)\right|\right)\right]^{p_{r s t}} \geq \beta+\frac{\epsilon}{2}\right) \geq \frac{\delta}{2}\right\}\right| \leq \frac{\epsilon}{2\left(d\left(\Delta_{\gamma}^{\alpha} x, y\right)\right)_{\Lambda^{3}}}+\frac{\delta}{2}$,

where $\sum_{u=1}^{\infty} \sum_{v=1}^{\infty} \sum_{w=1}^{\infty}\left|\gamma_{u v w} x_{u v w}\right|=0$, for all $r \geq u_{0}, s \geq v_{0}, t \geq w_{0}$. Further more, we can write $\left|\Delta_{\gamma}^{\alpha} x_{m n k}\right| \leq d\left(\Delta_{\gamma}^{\alpha} x_{m n k}, y\right)_{\Delta_{\gamma}^{\alpha}} \leq d\left(\Delta_{\gamma}^{\alpha} x, y\right)_{\Lambda^{3}}=d(x, y)_{\Delta_{\gamma}^{\alpha} x}$. For $r, s, t \geq u_{0}, v_{0}, w_{0}$

$\frac{1}{h_{r s t}} \sum_{(m n k) \in I_{r s t}} P\left(\left[f_{m n k}\left(\left|\Delta_{\gamma}^{\alpha} X_{m n k}\right|\right)\right]^{p}\right)=\frac{1}{h_{r s t}} P\left(\sum_{(m n k) \in I_{r s t}}\left[f_{m n k}\left(\left|\Delta_{\gamma}^{\alpha} X_{m n k}\right|\right)\right]^{p}\right)+$

$\frac{1}{h_{r s t}} P\left(\sum_{(m n k) \notin I_{r s t}}\left[f_{m n k}\left(\left|\Delta_{\gamma}^{\alpha} X_{m n k}\right|\right)\right]^{p}\right)<\frac{1}{h_{r s t}} P\left(h_{r s t}\left(\frac{\epsilon}{2}+\frac{\delta}{2}\right)+h_{r s t} \frac{\epsilon d(x, y)_{\Delta_{\gamma}^{\alpha} X}^{p}}{2 d(x, y)_{\Delta}^{p} \alpha}+\frac{\delta}{2}\right)=\epsilon+\delta . H e n c e$ $\left(x_{m n k}\right) \rightarrow\left(N\left(\Delta_{\gamma}^{\alpha}, p\right)_{\theta}\right)$

Corollary 5.1. If $\alpha$ be a proper fraction, $\beta$ be nonnegative real number, $f$ be an Musielak-Orlicz function and arbitary sequence $p=\left(p_{\text {rst }}\right)$ of strictly positive real numbers then the following statements are hold:

(i) $S \bigcap \Lambda^{3} \subset \Delta_{\gamma}^{\alpha}\left(S_{\theta}\right) \bigcap \Delta_{\gamma}^{\alpha}\left(\Lambda^{3}\right)$, (ii) $\Delta_{\gamma}^{\alpha}\left(S_{\theta}\right) \cap \Delta_{\gamma}^{\alpha}\left(\Lambda^{3}\right)=\Delta_{\gamma}^{\alpha}\left(w_{p}^{3}\right)$.

Theorem 5.2. Let $\alpha$ be a proper fraction, $\beta$ be nonnegative real number, $f$ be an Musielak-Orlicz function and arbitary sequence $p=\left(p_{\text {rst }}\right)$ of strictly positive real numbers. if $x=\left(x_{m n k}\right)$ is a $\Delta_{\gamma}^{\alpha}-$ triple difference rough lacunary statistically convergent sequence, then $x$ is a $\Delta_{\gamma}^{\alpha}$ - triple difference rough lacunary statistically Cauchy sequence.

Proof: Assume that $\left(x_{m n k}\right) \rightarrow\left(\Delta_{\gamma}^{\alpha}\left(S_{\theta}\right)\right)$ and $\epsilon, \delta>0$. Then $\frac{1}{\delta}\left|\left\{(m, n, k) \in I_{r s t}: P\left(\left[f_{m n k}\left(\left|\Delta_{\gamma}^{\alpha} x_{m n k}\right|\right)\right]^{p_{r s t}} \geq \beta+\frac{\epsilon}{2}\right)\right\}\right|$ for almost all $m, n, k$ and if we select $N$, then $\frac{1}{\delta}\left|\left\{(m, n, k) \in I_{r s t}: P\left(\left[f_{m n k}\left(\left|\Delta_{\gamma}^{\alpha} x_{N}\right|\right)\right]^{p_{r s t}} \geq \beta+\frac{\epsilon}{2}\right)\right\}\right|$ holds. Now, we have $\left|\left\{(m, n, k) \in I_{r s t}: P\left(\left[f_{m n k}\left(\left|\Delta_{\gamma}^{\alpha}\left(x_{m n k}-x_{N}\right)\right|\right)\right]^{p_{r s t}}\right)\right\}\right| \leq$ $\frac{1}{\delta}\left|\left\{(m, n, k) \in I_{r s t}: P\left(\left[f_{m n k}\left(\left|\Delta_{\gamma}^{\alpha} x_{m n k}\right|\right)\right]^{p_{r s t}} \geq \beta+\frac{\epsilon}{2}\right)\right\}\right|+$ 
$\frac{1}{\delta}\left|\left\{(m, n, k) \in I_{r s t}: P\left(\left[f_{m n k}\left(\left|\Delta_{\gamma}^{\alpha} x_{N}\right|\right)\right]^{p_{r s t}} \geq \beta+\frac{\epsilon}{2}\right)\right\}\right|<\frac{1}{\delta}(\beta+\epsilon)=\epsilon$, for almost $m, n, k$. Hence $\left(x_{m n k}\right)$ is a $\Delta_{\gamma}^{\alpha}$ - rough lacunary statistically Cauchy.

Theorem 5.3. If $\alpha$ be a proper fraction, $\beta$ be nonnegative real number, $f$ be an Musielak-Orlicz function and arbitary sequence $p=\left(p_{\text {rst }}\right)$ of strictly positive real numbers and $0<p<\infty$, then $N\left(\Delta_{\gamma}^{\alpha}, p\right)_{\theta} \subset \Delta_{\gamma}^{\alpha}\left(S_{\theta}\right)$.

Proof: Suppose that $x=\left(x_{m n k}\right) \in N\left(\Delta_{\gamma}^{\alpha}, p\right)_{\theta}$ and

$\left|\left\{(m, n, k) \in I_{\text {rst }}: P\left(\left[f_{m n k}\left(\left|\Delta_{\gamma}^{\alpha} x_{m n k}\right|\right)\right]^{p} \geq \beta+\epsilon\right)\right\}\right|$. Therefore we have

$\frac{1}{h_{r s t}} \sum_{(m n k) \in I_{r s t}} P\left(\left[f_{m n k}\left(\left|\Delta_{\gamma}^{\alpha} x_{m n k}\right|\right)\right]^{p}\right) \geq \frac{1}{h_{r s t}} \sum_{(m n k) \in I_{r s t}}(\beta+\epsilon)^{p} \geq$

$\frac{1}{h_{r s t}}\left|\left\{(m, n, k) \in I_{r s t}: P\left(\left[f_{m n k}\left(\left|\Delta_{\gamma}^{\alpha} x_{m n k}\right|\right)\right]^{p} \geq \beta+\epsilon\right)\right\}\right|(\beta+\epsilon)^{p}$.

So we observe by passing to limit as $r, s, t \rightarrow \infty$,

$\lim _{r s t \rightarrow \infty} \frac{1}{h_{r s t}}\left|\left\{(m, n, k) \in I_{r s t}: P\left(\left[f_{m n k}\left(\left|\Delta_{\gamma}^{\alpha}\left(x_{m n k}\right)\right|\right)\right]^{p} \geq \beta+\epsilon\right) \geq \delta\right\}\right|<$

$\frac{1}{(\beta+\epsilon)^{p}}\left(P\left(\lim _{r s t \rightarrow \infty} \frac{1}{h_{r s t}} \sum_{(m, n, k) \in I_{r s t}}\left[f_{m n k}\left(\left|\Delta_{\gamma}^{\alpha}\left(x_{m n k}\right)\right|\right)\right]^{p}\right)\right)=0$ implies that

$x \in \Delta_{\gamma}^{\alpha}\left(S_{\theta}\right)$. Hence $N\left(\Delta_{\gamma}^{\alpha}, p\right)_{\theta} \subset \Delta_{\gamma}^{\alpha}\left(S_{\theta}\right)$.

Competing Interests: The authors declare that there is not any conflict of interests regarding the publication of this manuscript.

\section{REFERENCES}

[1] A. Esi , On some triple almost lacunary sequence spaces defined by Orlicz functions, Res. Rev., Discr. Math. Struct., 1(2) (2014), 16-25.

[2] A. Esi and M. Necdet Catalbas,Almost convergence of triple sequences, Glob. J. Math. Anal., 2(1) (2014), 6-10.

[3] A. Esi and E. Savas, On lacunary statistically convergent triple sequences in probabilistic normed space, Appl. Math. Inf. Sci., 9 (5) (2015), 2529-2534.

[4] A. J. Datta A. Esi and B.C. Tripathy,Statistically convergent triple sequence spaces defined by Orlicz function , J. Math. Anal., 4(2) (2013), 16-22.

[5] S. Debnath, B. Sarma and B.C. Das ,Some generalized triple sequence spaces of real numbers, J. Nonlinear Anal. Optim., 6(1) (2015), 71-79.

[6] H. Kizmaz, On certain sequence spaces, Can. Math. Bull., 24(2) (1981), 169-176.

[7] P.K. Kamthan and M. Gupta, Sequence spaces and series, Lecture notes, Pure and Applied Mathematics, 65 Marcel Dekker, Inc. New York, 1981.

[8] J. Lindenstrauss and L. Tzafriri, On Orlicz sequence spaces, Israel J. Math., 10 (1971), 379-390.

[9] J. Musielak, Orlicz Spaces,Lectures Notes in Math.,1034, Springer-Verlag, 1983.

[10] A. Sahiner, M. Gurdal and F.K. Duden, Triple sequences and their statistical convergence, Selcuk J. Appl. Math. , 8 No. (2)(2007), 49-55.

[11] A. Sahiner, B.C. Tripathy, Some I related properties of triple sequences, Selcuk J. Appl. Math. , 9(2)(2008), 9-18.

[12] N. Subramanian and A. Esi, The generalized tripled difference of $\chi^{3}$ sequence spaces, Glob. J. Math. Anal., 3(2) (2015), 5460 . 